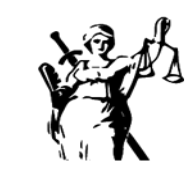

JUSTICIA

ISSN impreso $0124-744,1$
ISSN digital $2590-456$

\title{
El emprendimiento en Colombia: análisis del marco legal y su incidencia en la generación de empleo
}

\section{Entrepreneurship in Colombia: analysis of the legal framework and its impact on employment generation}

\author{
(iD) \\ William Alejandro Niebles Nuñez \\ Universidad de Sucre, Colombia \\ william.niebles@unisucre.edu.co
}

\author{
iD \\ Leonardo David Niebles Nuñez \\ Universidad del Atlántico, Colombia \\ leonardoniebles@mail.uniatlantico.edu.co
}

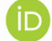 \\ Ignacio Ángel Barrios Parejo \\ Universidad del Atlántico, Colombia \\ profesorignaciobarrios@hotmail.com \\ Recibido: 07 de noviembre de 2019 / Aceptado: 11 de marzo de 2020 \\ https://doi.org/10.17081/just.25.38.4390
}

\begin{abstract}
Resumen
El objetivo de esta investigación es analizar el marco legal que se ha diseñado en Colombia para el emprendimiento, y observar cómo este impacta en la generación de empleo. La metodología utilizada fue de corte cualitativo con apoyo de la revisión documental, consultando y examinando diversos documentos y datos de corte científico como publicaciones, leyes, estudios de casos y artículos de revistas de corte académico. Para cumplir con los objetivos trazados se realizó una matriz de análisis bibliográfico que permitió filtrar los documentos seleccionados y extraer la información utilizada para este documento. Los resultados permiten visualizar los aspectos que son susceptibles de mejora y que pueden beneficiar el crecimiento del emprendimiento, renglón de vital importancia en el país, toda vez que genera el mayor número de empleos en la actualidad. La conclusión final sugiere oportunidades para el fortalecimiento del marco legal colombiano en lo relacionado con el emprendimiento, para así propiciar mayores oportunidades tanto en el ámbito social como económico.
\end{abstract}

PALABRAS CLAVES: Colombia, desarrollo social, emprendimiento, empleo, economía.

\section{Abstract}

The objective of this research is to analyze the legal framework that has been designed in Colombia for entrepreneurship, and to observe how this impacts on the generation of employment. The methodology used was qualitative with the support of documentary review, consulting and examining various documents and scientific data such as publications, laws, case studies and articles from academic journals. To meet the objectives set, a matrix of bibliographic analysis was carried out to filter the selected documents and extract the information used for this document. The results allow us to visualize the aspects that can be improved and that can benefit the growth of the enterprise, which is of vital importance in the country, since it generates the greatest number of jobs at present. The conclusion allows us to suggest opportunities for strengthening the Colombian legal framework in relation to entrepreneurship, in order to promote greater opportunities in both the social and economic spheres.

KEYWORDS: Colombia, entrepreneurship, employment, economy, social development.

\section{Como citar:}

Niebles, W., Niebles Nuñez, L. D., \& Barrios Parejo, I. A. (2020). El emprendimiento en Colombia: análisis del marco legal y su incidencia en la generación de empleo. Justicia, 25(38), 35-52. https://doi.org/10.17081/just.25.38.4390 


\section{Introducción}

El hombre es un ser de necesidades creadas y su evolución histórica es prueba de ello, pues cada uno de sus esfuerzos por avanzar en términos científicos, tecnológicos, económicos y sociales, entre otros, dan cuenta de su innato carácter innovador. Como parte de esa condición natural, los países están llamados a idear estrategias, políticas, y programas que vayan orientadas a estructurar un ambiente para las personas que están en la búsqueda de opciones que faciliten su crecimiento en todas las dimensiones inherentes al individuo (Kraus, Niemand, Halberstadt, Shaw y Syrjä, 2017). Es así como surge el término emprendimiento, el cual se encuentra asociado a una cantidad de factores con multiplicidad de aristas por examinar, y es justo el aprovechamiento de estas como oportunidades, lo que favorece la generación de conocimiento.

Para poder trazar una ruta de profundización en este tema y tomando en consideración los vertiginosos cambios del contexto global actual, se hace inevitable pasar por una revisión de cuál es el tratamiento que se le ha dado al emprendimiento, así como observar las tendencias tanto nacionales como internacionales. En este sentido la investigación por parte de los actores que integran el entorno de la educación superior, cumple un papel fundamental dentro de la sociedad, por ser los responsables de liderar los ámbitos de formación, fortalecimiento y promoción de buena parte de las personas que al final de sus programas académicos, podrán iniciar procesos de emprendimiento o insertarse a iniciativas relacionadas en sus áreas de interés.

De lo anterior se desprende la motivación de desarrollar una detallada observación en los avances que se han dado en Colombia, analizando los antecedentes del emprendimiento, teorías asociadas al fenómeno, verificar el marco legal vigente y también los datos de evolución del empleo, como efecto derivado de esta línea de desarrollo socio-económico a nivel nacional. Es relevante realizar este tipo de contrastes, ya que, el crecimiento social en todos los países se ve influido en gran medida por las oportunidades laborales o la dinámica en la creación de puestos de trabajo.

Así las cosas, el propósito de este documento es hacer un estudio del emprendimiento en Colombia, el marco normativo que lo contiene y analizar las cifras de empleo en relación a lo anterior como aspecto vital para toda economía. De igual forma, se revisan aportes de investigaciones y teorías relacionadas de autores diversos, buscando así, apreciar desde una visión de rigor el desarrollo de las iniciativas que buscan el favorecimiento del emprendimiento.

\section{Metodología}

El estudio se fundamenta en un enfoque cualitativo el cual es señalado por autores como Creswell y Creswell (2017), como un método que combina herramientas de reflexión y documentación, para comprender los fenómenos y hacerlos alcanzables a las partes de interés; así también, vincula técnicas basadas en el análisis de cualidades, tomando como referencia teorías, opiniones y manifestaciones similares de investigaciones previas.

Es de resaltar que los enfoques cualitativos persiguen exponer la realidad en su forma natural, sin reducirla a hechos numéricos en concreto y facilitando la observación de componentes inherentes a cada problemática investigada (Taylor, Bodgan y DeVault, 2015). En este sentido, se aborda la temática del emprendimiento desde una perspectiva socio-económica, analizando el marco legal en Colombia y aspectos asociados a la generación de empleo. 
Para recopilar la información, se utilizó la revisión de bibliografía reseñada por Chohan (2019), como una técnica para capturar información confiable de fuentes secundarias, con el propósito de construir nuevos enfoques o reflexiones. En este caso, se examinaron artículos científicos de corriente principal, publicados en bases de datos y repositorios de reconocida trayectoria a nivel nacional e internacional, entre estos se pueden mencionar: Scielo, Dialnet, Springer, Emerald, Redalyc, Scopus y Wos. Asimismo, se definieron criterios de búsqueda utilizando palabras clave asociadas con el tema; se realizó igualmente la selección de documentos en idioma inglés, delimitando la elección a los últimos siete años, con el fin de conformar un conjunto de títulos que orientaran de forma confiable el estado del arte del emprendimiento en Colombia, su marco legal y datos sobre el empleo.

\section{Resultados}

\section{Teorías sobre emprendimiento y su impacto en el desarrollo social}

El inicio del siglo XX fue una época particularmente importante para el emprendimiento, pues se comienzan a sentar las bases teóricas sobre las cuales, en años posteriores se fundamenta el concepto. Schumpeter (1934) es el primer autor que emite postulados concretos, creando una hipótesis en la que afirma que, los emprendedores son personas con una alta tolerancia al riesgo frente a la búsqueda de soluciones poco convencionales o desconocidas a las necesidades del entorno, probar estrategias nuevas para llevar a cabo actividades tradicionales y proporcionar al mercado alternativas novedosas.

Un elemento que llama la atención de los lineamientos propuestos por este autor, es que los individuos optan por emprender, centran su acción en la obtención de resultados exitosos como producto de sus ideas innovadoras, por lo que la generación de fortuna es solo el resultado de lo anterior. Es decir, el objetivo del emprendedor nace para resolver o aportar a una situación de su entorno, pero no siempre buscando un mero fin económico, aunque el resultado de su iniciativa usualmente deriva en este tipo de beneficios.

Partiendo de lo mencionado, se han desprendido nuevas investigaciones orientadas a dilucidar otros aspectos relacionados, como es el caso de Neto, Rodrigues y Panzer (2017), quienes basados en las premisas de gestión estratégica formuladas por Schumpeter, consideran que para entender cómo los futuros empresarios logran alcanzar el éxito comercial y económico de sus negocios, se deben determinar las técnicas que utilizan para agilizar la detección de oportunidades y captar los beneficios competitivos en el mercado.

No obstante, en muchos casos existe un divorcio entre ambos aspectos, es decir, compañías que localizan nichos desconocidos, pero no son capaces de generar elementos de valor que les haga sobresalir en el mercado o, por el contrario, que ostenten una posición comercial ideal, sin embargo, dejan a un lado la búsqueda de otras alternativas de desarrollo empresarial (innovación), bien sea para hacer propuestas inéditas o para solventar de una mejor manera situaciones convencionales (Molano y Campo, 2014).

Es importante poner de manifiesto, tal como lo hacen Castro, García y Adame (2015), quienes han sido guiados en parte por las teorías planteadas por Schumpeter, que un emprendedor no es necesariamente un empresario, y viceversa, pese a que sean similares. En el caso del primero, este es la persona que encausa su camino hacia la innovación, formando ideas que enfocan su pensamiento más allá de los límites establecidos para proponer soluciones revolucionarias. Entre tanto el segundo, se desempeña de mejor manera llevando a cabo procesos de corte administrativo, gerencial y de integración de recursos, por lo cual puede orientarse por estilos de liderazgos totalmente específicos (Palma, Rojas y Barrios, 2017). 
El emprendedor, también denominado por investigadores de tradición como el creativo imaginario, no siempre participa del proceso de estructuración de la empresa, como sí es el caso del segundo actor (empresa) que, de acuerdo con lo propuesto por Marshall (1890), pone en práctica sus conocimientos directamente en la compañía, aporta recursos económicos en la ejecución de las fases del proyecto, fundamenta su actuación en dirigir los elementos internos y externos de la organización, triunfando a través de los logros de la entidad.

Las dos ópticas presentadas (emprendedor y empresario) son igualmente válidas, además de necesarias, para impulsar lo discutido inicialmente respecto al aprovechamiento de la oportunidad e identificación de las ventajas competitivas, pues finalmente el objetivo común apunta al progreso económico de la sociedad en la cual se desenvuelve el innovador. En este sentido se despliega la investigación de autores como Boutillier y Uzunidis (2014), quienes proponen que tanto el emprendedor idealista, como el empresario pragmático pueden coexistir, ya que cada uno tiene un campo de acción dado por el contexto imperante en la región en un momento específico. Esto quiere decir que el individuo puede aprender a descubrir continuamente nuevos horizontes, pero al mismo tiempo debe contar con las herramientas necesarias para afrontar con aplomo las etapas de incertidumbre.

Como se puede apreciar, resulta difícil hablar de emprendimiento sin referirse por defecto a la competitividad, aspecto crucial para el desarrollo social y económico. Con referencia a lo anterior, se hace pertinente citar la teoría más representativa del tiempo reciente y planteada en la década de los 90 por el norteamericano Michael Porter. Dicha teoría define que el valor agregado, en cuanto a la producción de bienes o servicios que logra que un país tenga un índice de crecimiento económico más elevado que el de otro, lo otorga la generación continua de ideas que tienen acogida dentro y fuera de sus fronteras (Porter, 1990).

Como una herramienta de apoyo, el autor buscar dar una orientación precisa para evaluar los grados de competitividad presentes en el contexto empresarial, para lo cual desarrolló una metodología denominada las cinco fuerzas de Porter, que tiene como propósito precisar las mejores condiciones de rendimiento de un área productiva al establecer la distribución de los recursos monetarios obtenidos. Los cinco factores establecidos abordan la interacción entre compradores, vendedores, nuevos contendientes en el mercado, innovación en la manufactura y competencia entre los actores del sector (Porter, 1979). Es notorio que la teoría propuesta tiene más de 4 décadas de antigüedad, por lo que en la actualidad se ha cuestionado si estos preceptos aún siguen vigentes.

Es así que investigadores como Dälken (2014), se han dado a la tarea de comprobar este planteamiento, pues es bien sabido que el auge que ha tenido el desarrollo tecnológico ha generado cambios muy profundos en diversas áreas. Dentro de sus hallazgos este autor puntualiza que han surgido tres nuevas fuerzas que ciertamente han impactado significativamente en la estructura competitiva de la industria. No obstante, no existe alteración alguna en el esquema del modelo inicial, sino que las aristas recientemente identificadas influyen de forma simultánea en cada uno de los elementos del prototipo original.

En definitiva, tener la delantera en materia de mercados globales ha generado una importante atención a nivel internacional por varios años y, la incesante necesidad de describir su comportamiento, ha ocupado el tiempo de diferentes autores que buscan hacer aportes pioneros en el área. Así lo demuestra Wang (2014), quien indica que históricamente se han mantenido dos hipótesis de la competencia empresarial, una fundamentada en el mercado y la otra centrada en los recursos. Los avances recientes apoyan la influencia de factores como el conocimiento o el fortalecimiento de habilidades básicas, pero finalmente la mayor parte de los postulados se siguen orientando por la segunda tesis mencionada. 
De la misma manera, otros investigadores como Sölvell (2015), se han dedicado a estudiar y apoyar la obra de Michael Porter: La ventaja competitiva de las naciones (1990), los mismos indican que esta teoría sigue cada día más vigente, dando pie a que los grupos empresariales y los miembros del sector educativo sigan encaminados por esa misma ruta. De esta forma, los principios planteados generan nuevos puntos de vista que puedan dar origen a políticas públicas orientadas a propiciar la sana competencia comercial, además de la inclusión en los programas académicos de tópicos dirigidos específicamente a potenciar el aprendizaje de estrategias que garanticen una participación exitosa de los futuros profesionales en los mercados mundiales.

En el mencionado estudio, también se señala cómo haciendo una evaluación de la conducta económica de los países a nivel macroscópico, se pueden promover modernos patrones de competencia a menor escala que puedan perdurar en el tiempo. Destaca la trascendencia de una contienda saludable entre los participantes de la actividad comercial y adicionalmente se presenta la figura del clúster, que consiste en agrupaciones de empresas que se dedican al mismo sector de la economía, desarrollan su actividad en ubicaciones cercanas y coexisten en un ambiente de colaboración mutua. Ahora bien, para poder hacer un análisis apropiado del tema y ya teniendo una noción de las teorías desarrolladas al respecto, es necesario tomar como base para la discusión tres conceptos claves. El primero de ellos es el emprendimiento, término que tiene una connotación particular en Colombia pues implica poner en práctica el pensamiento crítico, además de implementar las acciones que se requieran para hallar alternativas a través de una perspectiva globalizada. Para esta tarea es imprescindible contar con líderes ecuánimes que tengan la habilidad de estimar y mitigar los riesgos asociados de índole tanto económico como social (Ley 1014, 2006).

El segundo factor a definir es la competitividad, que se concibe como la capacidad que posee un individuo, llámese país o empresa, de llevar al mercado un producto o servicio novedoso, que pueda satisfacer las necesidades de sus clientes, a la par de crear estrategias que le permitan alcanzar los objetivos propuestos, mantener la ventaja sobre las demás empresas del mismo sector económico y mejorar los procesos acordes con tendencias vigentes, respondiendo así no solo a las exigencias del entorno, sino también a las necesidades socio-económicas que atienden las naciones a nivel global (Porter, 1990). En los años recientes, autores como Pinzón (2016) complementan la teoría citada indicando que existen unas variables exógenas que consolidan el protagonismo del Estado en la detección de puntos críticos que ameriten la generación de condiciones óptimas y otras de tipo endógeno que demandan que las compañías consigan amalgamarse a las nuevas circunstancias, para así hacerse sustentables y permanecer en el tiempo a pesar de las variaciones y condiciones de cada momento.

El tercer componente necesario para el desarrollo del escenario analizado es la educación superior en la figura de las universidades, interpretada por Rangel, Rubiano y Riaga (2015) como un organismo facultado para generar, divulgar y compartir conocimientos por medio de la investigación y que en los últimos años, ha sufrido cambios sustanciales que actualmente la ubican como uno de los pilares de la nueva economía, pues brinda las herramientas formativas para el desarrollo de habilidades competitivas que marcan la diferencia con el resto de los integrantes del sector emprendedor, al tiempo que aporta estrategias en procura de su sustentabilidad (Cáceres, 2017).

\section{Antecedentes Internacionales y nacionales sobre emprendimiento}

El acelerado avance de la tecnología, además de la incesante evolución de las culturas a nivel mundial, ha traído como consecuencia que cada nación trabaje constantemente en lograr una evolución integral. Tal es el caso que plantea Mok (2015) en su artículo Transformaciones de la educación superior para la competitividad mundial: Respuestas de política pública, consecuencias sociales e impacto en la profesión 
académica en Asia, quien expone como el crecimiento de la industria del conocimiento ha creado la necesidad de hacer transformaciones de fondo para la promoción de la capacidad emprendedora con apoyo de las políticas públicas.

La metodología utilizada para la investigación se basó en un análisis bibliográfico de las diversas estrategias que los estados y las universidades del continente asiático han implementado en la renovación del proceso de innovación y creación de empresa. El resultado deja entrever un robusto inventario de las medidas políticas, sociales y académicas relevantes que se han aplicado en este tema, partiendo de los más pequeños actores, hasta llegar a los más robustos en cada renglón económico del continente mencionado.

Por otra parte, autores como Guerrero, Urbano y Fayolle (2016), examinan la temática en su publicación Actividad empresarial y competitividad regional: pruebas de las universidades empresariales europeas, donde ponen de manifiesto que la sinergia entre la dinámica corporativa y la libre competencia entre las regiones, se ha convertido en un importante foco de interés en los ámbitos políticos y educativos, pues está comprobado que la vinculación de la academia con los procesos productivos asegura el progreso económico de los países de forma sustentable e integradora.

Se utilizó por parte de los autores citados, un enfoque de investigación cuantitativo con un diseño documental y de campo para estudiar los datos de 102 universidades pertenecientes a doce países de Europa, con el propósito de evidenciar cómo la contribución de las instituciones de educación superior aporta herramientas cognitivas al desarrollo de las empresas que a su vez, se proyectan en el auge financiero de la nación. Derivado de este planteamiento, resultó que los programas asociados a las políticas públicas, dieron buenos resultados al integrarse con las iniciativas emprendedores derivadas de la academia y que estas se hicieron más competitivas gracias a los recursos procedentes de los programas oficiales.

De igual forma, en la publicación de Aleksejeva (2016), denominada Competitividad y sostenibilidad del país: impacto en la educación superior se expone que, en la actualidad el conocimiento ha adquirido un mayor valor, por lo que es considerado la fuente primaria de la que se desprenden los procesos creativos y debido a esto, las grandes potencias internacionales han asignado una cantidad significativa de recursos para la promoción de políticas y programas en favor de la educación, pues opinan que es un factor clave para garantizar la sustentabilidad y el progreso tanto económico como social de la población.

Haciendo uso de una metodología comparativa/cuantitativa, esta investigación buscó precisar cuál ha sido la incidencia de la formación profesional de los ciudadanos en el crecimiento monetario de los países bálticos y nórdicos, asegurándoles una competitividad sostenible en el exterior. A raíz de este estudio, se concluye que es fundamental que haya un marco legal integrador, donde desde el Estado se impulse una ayuda mutua entre el empresariado y las universidades, a fin de facilitar el intercambio de ideas en beneficio de un objetivo común.

Entre tanto, investigadores como los españoles Torres-Valdés (2015), en su artículo Estímulo a la creatividad y emprendimiento joven para la innovación social en un contexto global, cuyo propósito fue incentivar a la nueva generación para la puesta en marcha de su capacidad e inventiva, en pro de generar soluciones que fomenten la evolución de la sociedad de forma sostenible y perdurable, se deja entrever el papel fundamental del Estado, toda vez que se emiten los estímulos correctos, los resultados se dan de manera destacable.

El método utilizado por los investigadores fue la verificación sistemática de textos acerca de las diferentes perspectivas del tema, y a partir de allí, se obtuvo como resultado la formulación de procedimientos desde las instituciones, los cuales permiten orientar eficientemente al público meta y aprovechar las 
políticas públicas en cada contexto. La participación de estas políticas se definió como un rol impulsador, pues el marco legal analizado contó con las bases necesarias para facilitar la actividad emprendedora en el país de análisis.

Lo anterior también encuentra sustento en autores ecuatorianos como Rivera y Rodríguez (2015), en el documento Educación para el emprendimiento, donde el objetivo se centra en exaltar el auge internacional de alternativas innovadoras por medio de las cuales se pueden satisfacer las crecientes necesidades de las comunidades, así como también se busca enaltecer el papel de la educación como factor de impacto, tanto en el individuo como en las acciones relacionadas con su formación, y por consiguiente en su entorno socio-económico. A nivel metodológico se recurre a la revisión de las fuentes bibliográficas relacionadas con el tópico en cuestión en bases de datos científicas. Al final la información recabada permite apreciar que, existe la necesidad de ahondar en la investigación, como proceso que contribuya con la valoración y la estructuración de propuestas con mayor viabilidad, aprovechando así las alternativas propuestas desde los ámbitos públicos y privados.

Continuando el recorrido de revisión de experiencias de emprendimiento en otros países, se observa en la exploración de González, Jenkins y Lloret (2016), que lleva por nombre Desafíos para la investigación académica de negocios en América Latina, el análisis entre la dupla innovación y desarrollo, los cuales se han convertido en un vehículo fundamental para impulsar la creatividad empresarial, incrementar la competencia intercompañías y alcanzar el ascenso económico de las naciones, al mismo tiempo que asegura la prosperidad de la población.

Con la ayuda del método cuantitativo, se realizó una evaluación de la conducta reportada por los países de América Latina con respecto a los términos centrales de la investigación, obteniendo como resultado que no existe suficiente apoyo financiero para apalancar las nuevas ideas, y la colaboración efectiva es destinada a propuestas muy puntuales, por lo cual los marcos legales son susceptibles de revisión y mejora. Por lo antes mencionado, los autores le atribuyen un rol protagónico a las escuelas de negocios para lograr que se generen nuevos hallazgos en la materia, aplicados a escenarios concretos y además, se sugiere la renovación de políticas en esta dirección.

Siguiendo el hilo conductor del documento, los académicos Rubach, Bradley y Kluck (2015), comentan acerca de un factor característico de las iniciativas empresariales que se generan en el continente americano, en su artículo La necesidad de emprender: un estudio latinoamericano, que consiste en analizar el emprendimiento derivado o puesto en marcha por necesidad, cuya opción es tomada generalmente por los inmigrantes y los latinos que buscan solventar su situación económica, además de ser una alternativa para abandonar el empleo tradicional.

Mediante la metodología cualitativa y utilizando datos extraídos del Global Entrepreneurship Monitor (GEM), se determina que los futuros empresarios aplican este formato en países como Estados Unidos, pero que lamentablemente, encuentran un número importante de dificultades en los organismos que se encargan de la formalización de la actividad comercial, por lo que se proponen algunos canales para mitigar esta situación, ya que finalmente estos aportes también coadyuvan al progreso del país.

Como otro ejemplo de los avances en el tema a lo largo de América Latina se observa el documento titulado Prácticas de innovación en las economías emergentes: ¿lmportan las asociaciones universitarias?, donde los autores Guerrero, Urbano y Herrera (2019) buscan dejar en evidencia el importante rol que desempeñan las integraciones estado/empresa/universidad, en su constante proceso generador de conocimiento y en la evolución de las economías nacientes, colocando especial atención en el caso mexicano. 
El estudio realizado es de corte cuantitativo, cuyos datos han sido extraídos del Instituto Nacional de Estadística y Geografía de México, obteniendo resultados que dan referencia sobre la importancia de la intervención académica, tanto en la fase exploratoria como en la gestión de subsidios derivados de políticas públicas para el financiamiento de los proyectos.

Por otro lado, como parte del esfuerzo por conocer qué es lo que motiva a una persona a encaminarse por un proyecto propio, también se ha indagado en investigaciones como la de Krauss, Bonomo y Volfovicz (2018), denominada Modelo predictivo de la intención emprendedora universitaria en Latinoamérica. Este artículo aborda cómo a través de la técnica de regresión ordinal logística, se logra estructurar una fórmula matemática, que pronostica la inclinación de un alumno de educación superior a llevar a cabo un emprendimiento.

Como resultado de esta exploración, que contó con la participación de estudiantes de nueve distintas universidades de Latinoamérica, y que poseen como principales factores de influencia aspectos como programas gubernamentales, vínculo con iniciativas familiares, creatividad, motivación y actitud, se obtiene que las personas provenientes de países como Ecuador, Colombia y México tienen una probabilidad mayor de encaminarse por tendencias innovadoras que los individuos originarios de naciones como Brasil, Chile y Uruguay.

Así las cosas, Sánchez, Ward, Hernández y Flórez (2017), exponen su punto de vista en el trabajo Educación emprendedora: Estado del arte, en el cual se analiza la inestabilidad laboral como situación común en América Latina y la manera en que esto induce a sus ciudadanos a tomar partido por opciones como el autoempleo, además de la generación de alternativas que sean sinónimo de crecimiento económico para la sociedad. Se aplicó una metodología documental que incluyó la recolección de información de fuentes científicas, textos y publicaciones de revistas.

Los resultados del estudio, ponen en evidencia que a pesar de que existe un abanico de opciones que apoyan la formación integral y consolidación de los proyectos empresariales, los países latinoamericanos requieren de un esfuerzo adicional para sentar bases más sólidas en las instituciones de educación superior, específicamente a nivel curricular en materia de emprendimiento, pero también del soporte gubernamental para lograr programas y políticas públicas ajustadas a cada realidad.

Entre tanto, a nivel nacional también se ha indagado sobre el contexto del emprendimiento, encontrando avances en trabajos como los de Melo y Villa (2015), en la publicación del Banco Interamericano de Desarrollo (BID), Panorama actual de la innovación social en Colombia. Dicha publicación tuvo por propósito ofrecer un punto de referencia de cómo se proyecta la labor de los diferentes actores públicos y privados en pro de fomentar en la población la creación de iniciativas, a través de una metodología documental de identificación de programas, planes y políticas públicas que contribuyan con el logro de los objetivos emprendedores.

El resultado obtenido es una importante lista de propuestas que apalancan el crecimiento y desarrollo de áreas con oportunidades potenciales, además de estructurar nuevas fuentes de apoyo en formación y financiamiento, entre otros. En la tabla 1 se muestran los porcentajes de las ideas de negocios que se han visto beneficiadas por las convocatorias del Centro de Innovación Social (CIS), institución creada por el Estado colombiano, como parte de una serie de estrategias públicas direccionadas a propiciar programas con orientación social que impulsen soluciones creativas a las dificultades de las comunidades. 
TABLA 1. CONVOCATORIAS DE INNOVACIÓN ABIERTAS DEL CIS

\begin{tabular}{|l|c|}
\hline Convocatorias & Porcentaje de propuestas apoyadas \\
\hline Ideas para el cambio: agua y pobreza & $18,03 \%$ \\
\hline Proyecta Colombia & $3,67 \%$ \\
\hline Genera & $6,50 \%$ \\
\hline Buen provecho & $2,70 \%$ \\
\hline Vía láctea & $3,45 \%$ \\
\hline Socialapp & $2,67 \%$ \\
\hline Ideas para el cambio: Pacífico pura energía & $24,61 \%$ \\
\hline
\end{tabular}

Fuente: Adaptado de la publicación del BID, 2015

La relevancia de estos datos pone de manifiesto la importancia que le está dando el BID a las acciones que están poniendo en marcha países como Colombia respecto a la denominada innovación social, lo cual se puede observar en las propuestas relacionadas con energía, agua y pobreza, que han recibido el porcentaje más alto de apoyo económico para su ejecución y que hacen parte de iniciativas globales que también se replican en otros países del área.

De igual modo, en el Modelo de formación en emprendimiento social para Instituciones de Educación Superior en Colombia (Vega y Mera, 2016), se definen los elementos que componen el prototipo de adiestramiento que se imparte a los futuros empresarios desde las universidades, acorde con las características del mercado nacional. En este estudio, la metodología empleada es de tipo exploratoria y de estudio de diversos contenidos, para lograr una posterior clasificación de la información que conlleve a esbozar una nueva propuesta de enseñanza. A partir de esto se desprende un esquema que concentra al individuo como eje central de la innovación, luego está el proyecto que desea sacar a flote y por último el ámbito donde se plantea la iniciativa emprendedora interactuando en este escenario con las políticas y programas oficiales de cada región. También es preciso mencionar estudios como los de Gutiérrez y Baquero (2017) que han realizado su aporte en la materia con la publicación Nueva propuesta transversal de emprendimiento e innovación en los programas educativos en la educación de tercer nivel (terciario). En esta publicación, los autores indican que la sociedad moderna precisa del conocimiento y del apoyo estatal, para dar soluciones a las diversas necesidades que manifiesta la población. Esto trae consigo una serie de procesos que solamente es posible llevarlos a cabo con el impulso que genera el denominado espíritu empresarial, que puede ser innato en la persona, pero que también puede ser aprendido por el individuo y favorecido por las políticas públicas.

A partir de una revisión documental de algunos programas académicos de varias instituciones de educación superior de Bogotá, se determina que a pesar de la transversalidad que se le ha dado al emprendimiento en las carreras universitarias, no ha sido exitosa la aplicación de los conocimientos en los escenarios reales, por tal motivo, los autores plantean un prototipo que permite al estudiante descubrir y potenciar sus habilidades en el campo, asi como integrar aspectos derivados de iniciativas del gobierno para tal fin. Seguidamente, en el texto titulado Emprendimiento e innovación para impulsar la competitividad desde la universidad en la ciudad de Barranquilla (Colombia), cuyo estudio es abordado por RenízGarcía y Rojas-Millán (2018), se explica cómo se logra la integración de las Tecnologías de la Información y la Comunicación (TIC) con los procesos creativos en el área de la salud a través del llamado living-lab, el cual consiste en una técnica colaborativa en la que los usuarios, a partir de sus experiencias, son partícipes de la formulación de soluciones al respecto. 
Partiendo de una metodología documental, se evidencian los progresos experimentados como resultado de la interacción con el Laboratorio Viviente, además de constatar la positiva acogida que ha tenido desde los diferentes sectores, educativo, gubernamental y empresarial principalmente, por lo que se estima oportuno proseguir con su uso en beneficio de la región, integrando las partes citadas como base para un crecimiento sostenido en la ruta emprendedora. Como se puede apreciar, las investigaciones recientes dan cuenta de resultados diversos que muestran como parte integral de los ecosistemas emprendedores, el papel del Estado como componente fundamental para la dinámica forjadora de creación de nuevas empresas. Autores como Baquero, Hernández y Saboga (2019) en su artículo La formación de emprendedores sociales desde algunos programas de Contaduría Pública, también describen de qué manera se ha evidenciado la contribución de las instituciones de educación superior en la preparación de los futuros egresados de este programa con orientación hacia los intereses de la comunidad con mediación de las políticas públicas.

Derivado de la implementación de un método documental, se analizan los programas y políticas públicas canalizadas hacia la innovación comunitaria, además de estudiar rasgos distintivos de las personas que se encaminan por esta opción, pertenecientes a la carrera de contaduría de tres centros universitarios del departamento de Cundinamarca. Luego del recorrido de la literatura, relacionado con el emprendimiento en el contexto nacional e internacional, se procede a abordar el marco legal del contexto colombiano, el cual sin lugar a dudas se aprecia como un componente definitivo al tiempo que contempla la variable de empleo como un efecto directo de todo el proceso.

\section{Marco legal del emprendimiento en Colombia}

Para lograr un análisis legal respecto del emprendimiento en Colombia, lo primero que se debe visualizar es el enfoque desde el punto de vista constitucional, ello con el fin de armonizar de manera global un escenario que ofrezca una clara dimensión del tema que se pretende ahondar y así verificar bajo qué derroteros se ha manejado el tema en el país. Esto permitirá definir si se está en un ambiente plenamente definido, o por el contrario predomina un ámbito susceptible de revisión o fortalecimiento. Por lo antes indicado, es necesario que dentro de los argumentos a presentar, se realice un acercamiento sobre la situación del emprendimiento en otros países, para poder tener un panorama más cercano a la realidad y así reflexionar sobre qué tan fácil o complejo es hacer empresa en Colombia, brindando argumentos de valor a quienes hoy desean empezar por esta senda. Generar conocimiento en relación al emprendimiento será siempre un aporte esencial para la promoción de una economía más autosostenible.

En el Título XII de nuestra Carta Magna (Constitución Política [CP]) 1991 se presenta lo relativo al régimen económico y de la Hacienda Pública y en su Capítulo I que corresponde a las disposiciones generales desarrolladas en el Artículo 333, que consagra: "La actividad económica y la iniciativa privada son libres, dentro de los límites del bien común. (...) La libre competencia económica es un derecho de todos que supone responsabilidades. La empresa, como base del desarrollo, tiene una función social que implica obligaciones. (...)." En concordancia con el artículo citado, se encuentra también en dicho compendio que el derecho al trabajo, es de aquellos de raigambre fundamental que debe cumplir una función social (Silva y Bermúdez, 2020), de tal manera que debe ser protegido por el Estado, obviamente esto requiere que la actividad ejecutada corresponda a una actividad lícita (Art. 25 C.P.). Bajo este escenario, queda claro que desde el punto de vista constitucional hacer empresa en Colombia es una prioridad, dada la relevancia que representa para el fomento del desarrollo integral a título social y económico. Desde el punto de vista legal y jurisprudencial se cuenta con un buen número de normatividades con base en las cuales se ha buscado ofrecer apoyo a los empresarios para la creación y formalización de las empresas, sin embargo, dichos sumarios no han sido suficientes y aún hoy, se sugiere por parte de agremiaciones, universidades y el mismo 
sector productivo, que los pequeños empresarios no cuentan con un escenario adecuado para poder hacer empresa, debido a los altos costos que implica adentrarse en dicho sentido.

A continuación, en la tabla 2 se presenta el recorrido normativo que aplica para el emprendimiento en el país.

TABLA 2. MARCO NORMATIVO PARA EL EMPRENDIMIENTO EN COLOMBIA

\begin{tabular}{|c|c|}
\hline NORMATIVIDAD & CONTENIDO \\
\hline Ley 344 de 1996 & $\begin{array}{l}\text { Se adoptan medidas tendientes a racionalizar y } \\
\text { minuir el gasto público, garantizar su financiamiento y } \\
\text { nar recursos hacia sectores deficitarios de la actividad }\end{array}$ \\
\hline Ley 550 de1994 & $\begin{array}{l}\text { Establece un régimen que promueva y facilite la reactivación empresari- } \\
\text { al y la reestructuración de los entes territoriales para asegurar la función } \\
\text { social de las empresas y lograr el desarrollo armónico de las regiones. }\end{array}$ \\
\hline Ley 789 de 2002 & $\begin{array}{l}\text { Por la cual se dictan normas para apoyar el empleo y ampliar la protec- } \\
\text { ción social y se modifican algunos artículos del Código Sustantivo de Trabajo. }\end{array}$ \\
\hline Decreto 934 de 2003 & $\begin{array}{l}\text { Se reglamenta el funcionamiento del Fondo Emprender (FE), el cual será adminis- } \\
\text { trado por el SENA y cuyo objeto exclusivo será financiar iniciativas empresariales. }\end{array}$ \\
\hline Ley 9 & $\begin{array}{l}\text { Por medio de la cual se modifica la Ley } 590 \text { de } 2000 \text { sobre promoción } \\
\text { del desarrollo de la micro, pequeña y mediana empresa colombiana. }\end{array}$ \\
\hline Ley 1014 de 2006 & a la cultura del emprendimiento \\
\hline $\begin{array}{l}\text { Sentencia C - } 392 \text { de } \\
2007\end{array}$ & 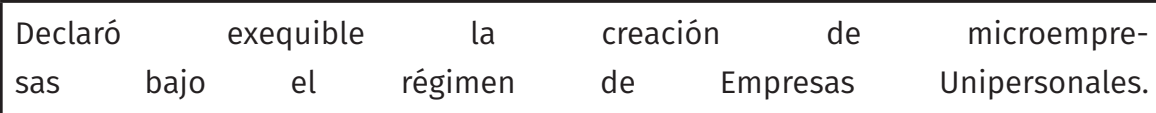 \\
\hline $\begin{array}{l}\text { Sentencia C - } 448 \text { de } \\
2005\end{array}$ & $\begin{array}{l}\text { Declaratoria de inexequibilidad de las expresiones "con concepto pre- } \\
\text { vio favorable de la Dirección de Apoyo Fiscal del Ministerio de Hacien- } \\
\text { da" contenidas en el artículo } 42 \text { de la Ley } 590 \text { de } 2000 \text { tal como quedó mod- } \\
\text { ificado por el artículo } 21 \text { de la Ley } 905 \text { de } 2004 \text { "por medio de la cual se } \\
\text { modifica la Ley } 590 \text { de } 2000 \text { sobre promoción del desarrollo de la micro, } \\
\text { pequeña y mediana empresa colombiana y se dictan otras disposiciones". }\end{array}$ \\
\hline $\begin{array}{l}\text { Código de Comercio } \\
\text { (1971) }\end{array}$ & $\begin{array}{l}\text { Permite conocer de manera clara las distintas empresas que se } \\
\text { pueden constituir en Colombia como las: Anónimas, colectivas, en Co- } \\
\text { mandita Simple o por Acciones, Limitadas, Extranjeras, de Hecho. }\end{array}$ \\
\hline Ley 1258 de 2008 & Por medio de la cual se crea la Sociedad por Acciones Simplificada. \\
\hline
\end{tabular}

Fuente: elaboración propia, 2020 (con base en normas expedidas en Colombia)

De acuerdo con el marco descrito, existen diversas posibilidades para crear empresas, conforme a las necesidades de cada persona, incluso existiendo la opción de hacerlo de manera individual o agrupando un determinado número de accionistas. En el caso de la modalidad más reciente incorporada y denominada Sociedad por Acciones Simplificadas (SAS) a modo de ejemplo, al no existir un límite de personas, se puede realizar la venta de acciones sin que jurídicamente estén obligados a permanecer en el tiempo, cuando en dado caso existan diferencias irreconciliables entre sus asociados lo que facilita su continuidad (Villarreal, 2017). 
De igual manera, esta modalidad societaria facilita la puesta en marcha y constitución, toda vez que se puede realizar por documento privado, lo que implica que el simple registro ante la Cámara de Comercio permite su accionar válidamente, pues solo se necesita un documento público cuando haya inscripción de bienes inmuebles, esto hace que, si se trata de empresarios sin mayores recursos, pueden poner en funcionamiento su empresa sin mayores formalismos, ofreciendo con ello una opción rápida y eficaz (Trujillo y Estrada, 2017). Siguiendo con la reseña de esta figura favorecedora del emprendimiento, en una SAS no se exige un término de duración en el tiempo, lo que facilita su ejecución de manera indefinida; no requiere un objeto social específico, lo único que se necesita como se había indicado líneas atrás es que la actividad desarrollada sea lícita, no es necesario contar con una revisoría fiscal o con una junta directiva lo que la hace más amigable en cuanto al tema de los costos de constitución, por lo que solo dependerán del capital suscrito y de los activos, así las cosas, se considera que este modelo ha impulsado la creación de empresas en el país en la última década (Abdala, 2018).

Al analizar la dinámica emprendedora a nivel internacional y con el ánimo de contrastar con el contexto colombiano, es pertinente citar el informe Doing Business 2016, donde se especificó que en Colombia para iniciar un negocio se demandan significativos desafíos, determinando que en un puntaje que tiene como base 11 indicadores, entre ellos la facilidad para iniciar una empresa, obtención de créditos y pago de impuestos, se obtuvo como resultado que el país logró el puesto 54 entre 189 países analizados (Durango y Rueda, 2020). De acuerdo con este análisis vemos que Colombia ha incrementado sus esfuerzos con miras a promover el escenario emprendedor, teniendo ya avances destacables y de reconocimiento a nivel internacional. La necesidad imperiosa de apoyar a las nacientes empresas y sobre todo buscar la manera de formalizar el empleo en Colombia, llevó al Estado a organizar una política de formalización empresarial en enero de 2019, la cual quedó estructurada a través del Consejo Nacional de Política Económica y Social 3956 (CONPES), formado en conjunto con el Departamento Nacional de Planeación, los Ministerios de Hacienda y Crédito Público, Salud y Protección Social, Trabajo, Comercio, Industria y Turismo y el Departamento Administrativo Nacional de Estadística (Rubiano, 2020).

Dentro del resumen ejecutivo del mencionado CONPES se indica que ha sido una necesidad del gobierno nacional en conjunto con las autoridades locales, gremios y entidades privadas, realizar grandes esfuerzos para reducir la informalidad empresarial, debido a los altos índices de esta misma presentados en Colombia. Alrededor del $75 \%$ de las microempresas en el país no están registradas (No cuentan con registro mercantil y tampoco con Registro Único Tributario [RUT]) y los niveles de cumplimiento en cuanto a contratación formal de trabajadores, pago de prestaciones sociales, implementación de una contabilidad formal, declaración y pago de impuestos son realmente bajos (Salcedo, Moscoso y Ramírez, 2020).

Al abordar el tema emprendedor en países con mayor crecimiento en la materia, se tiene que economías como la de Alemania, Japón, Estados Unidos, Reino Unido y Suiza, han logrado avances y gran posicionamiento gracias a la dinámica pública para la generación de marcos legales flexibles y enfocados en cada contexto; en Alemania por ejemplo, se tienen tiene altos estándares de cultura empresarial en lo relacionado con ética, leyes y regulaciones, por lo cual todos los actores concurren de manera armonizada en el fortalecimiento, educación e innovación de rutas de emprendimiento (Pazmiño, 2019). Por su parte en Japón, se puede destacar que existe un gran volumen de incubadoras, fondos para nuevas empresas y canales de contactos entre los emprendedores y el sector público/privado, a fin de combinar el Estado, la sociedad y las universidades, con el fin de lograr resultados en el corto tiempo. Así también, se puede mencionar que, en Estados Unidos a diferencia de muchas otras culturas, cuando un emprendedor no logra su objetivo inicial, es llevado a un programa de atención, donde la experiencia negativa es tomada como base para una nueva iniciativa, por lo cual se gestan procesos en cadena que usualmente en los segundos intentos generan resultados de alto impacto (Tamayo y Alvear, 2018). 
Así también se encuentra la experiencia del Reino Unido donde el papel más protagónico es el del gobierno, donde el Estado pone a disposición de las partes interesadas, numerosos recursos en línea, préstamos iniciales y plantillas gratuitas para hacer planes de negocios respaldados por entidades sin ánimo de lucro, que fomentan tanto los aspectos educativos como los de innovación e investigación (Correa, 2019).

\section{Cifras del emprendimiento y el empleo en Colombia}

A nivel latinoamericano, Colombia se considera como uno de los países con economía fuerte, ocupando el cuarto lugar de desarrollo en el año 2012, luego de países como Brasil, México y Argentina. El país se destaca en la industria del petróleo, el carbón, la minería en general y los productos agrícolas (Estrada y Uribe, 2013). La economía de Colombia se ha catalogado como fuerte, debido a que depende de diferentes industrias, productos y servicios, en el que nuevos emprendimientos han sido la respuesta para la lucha contra el alto desempleo y lograr así una posición fortalecida. Ciudades estratégicas como Medellín, Barranquilla y Bucaramanga, trabajan para la disminución de la brecha creada por el desempleo en aspectos fundamentales como oportunidad, acceso y recursos de apoyo, mediante la promoción de programas del gobierno a nivel regional y distrital, de la mano con asociaciones y entidades de carácter público y privado que promuevan la investigación, el desarrollo de emprendimientos y la fluctuación de negocios para la generación de pequeñas y medianas empresas (pymes) (Estrada y Uribe, 2013).

De acuerdo a cifras del Ministerio de Trabajo, las pymes representan más del 90\% del sector productivo, y generan empleo a más de 16 millones de colombianos (Mintrabajo, 2019). A continuación, se muestran las cifras que relacionan el comportamiento histórico del país en aspectos fundamentales como lo son emprendimiento y desempleo, lo cual explica el desarrollo de la economía y la variable de empleo como un determinante en el crecimiento del país. Es así, que al analizar las cifras del periodo 2008 - 2015, se encuentra que disminuyó la cantidad de personas emprendedoras nacientes, es decir, se redujo el interés en la población colombiana para la creación de nuevas empresas, en especial en el año 2014, que presentó el menor avance con un $24 \%$ con relación a otros años como 2012, 2013 y 2015, cuyas cifras fueron de $27 \%$, $29 \%$ y $28 \%$ respectivamente (Pinzón, 2019), lo anterior causó un impacto de forma directa en los años siguientes en la generación de empleo, ver seguidamente figura 1.

Figura 1. Emprendimiento y Desempleo en Colombia (2016-2018)

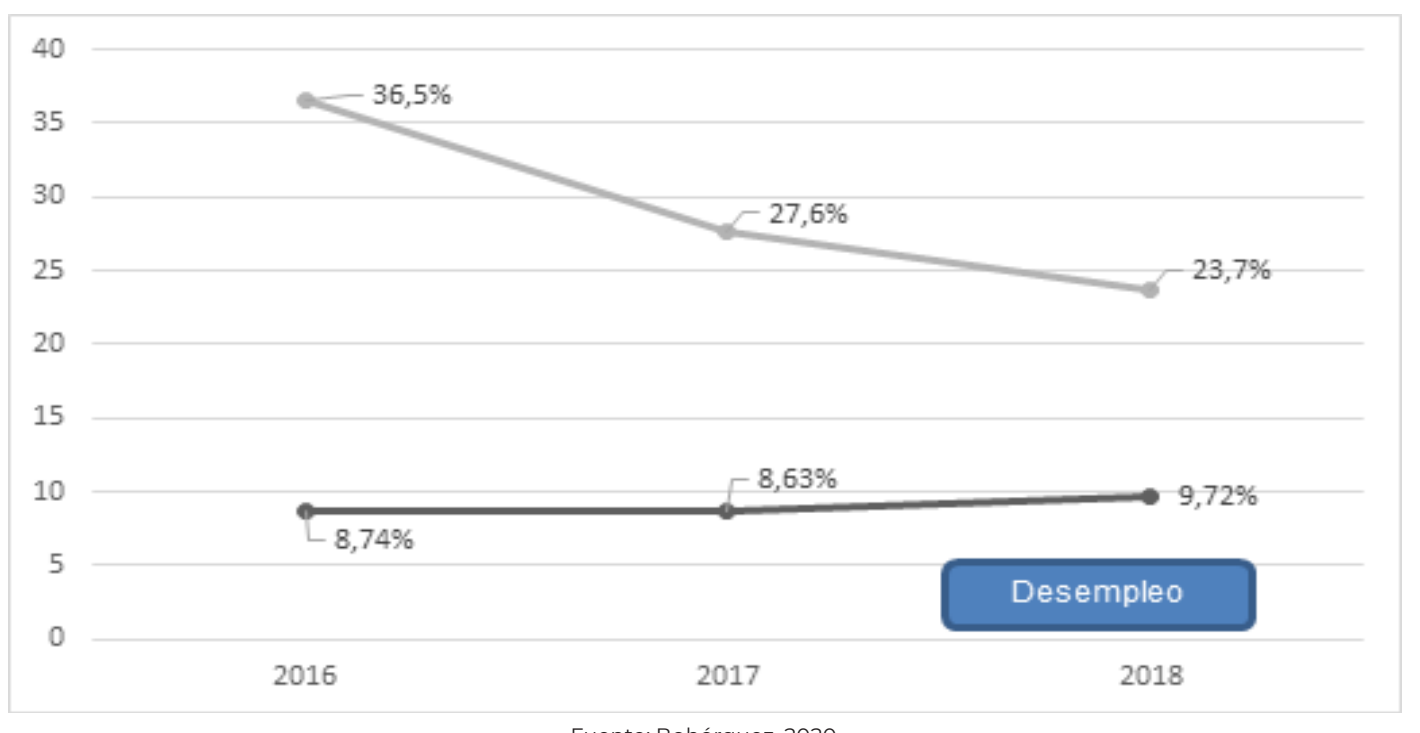

Fuente: Bohórquez, 2020 
Como se puede observar en la figura 3, y en relación a los años 2016, 2017 y 2018, hubo un aumento en el porcentaje de desempleo de casi el $1 \%$ y el número de emprendimientos a nivel nacional decayó en un 12.8\%. La disminución de empleabilidad en el país para el año 2018 fue representativa, alcanzando en total el 9.72\% como lo indica el Departamento Administrativo Nacional de Estadísticas (DANE, 2020). Los municipios que encabezaron la mayor acumulación de desocupados fueron: Buga y Buenaventura, y ciudades principales como Quibdó, Cúcuta y Armenia (Bohórquez, 2020).

Según reportes oficiales, la tasa de desempleo en 2019 aumento aún más alcanzando un 10,4\%, presentando una variación de $0,7 \%$; sin embargo, el sector que mantuvo en el país mayor empleabilidad fue el de la construcción con un $6.8 \%$; en cargos por posición ocupacional en el año 2019, los que generaron mayor empleabilidad fueron los jornaleros y empleados domésticos con 4.3 y $2.6 \%$ respectivamente (DNP, 2019).

\section{Discusiones}

Entre los aspectos que se rescatan luego del proceso de revisión y análisis de la información se destacan: Colombia es uno de los países de América Latina que muestra una importante inclinación hacían el desarrollo emprendedor, lo cual queda evidenciado por las cifras reportadas por organismos de observación a nivel global. Adicionalmente se valora que, en materia normativa el país ha dado pasos concretos para promover el desarrollo de las iniciativas emprendedoras, encontrando en la actualidad diversas figuras para propiciar el nacimiento de nuevas empresas.

No obstante, al realizar contrastes con países más avanzados en la materia, se estima que existen todavía oportunidades por explorar, pues si bien los programas derivados de la gestión oficial han dado ya resultados de reconocimiento, las cifras dadas a conocer por el DANE, permiten visualizar que a medida que se generan decrecimientos en los procesos de emprendimiento, se incrementa el porcentaje de desocupados, aspecto que puede ser intervenido mediante un mayor acompañamiento de las partes de interés (Estado/Universidad/Sociedad).

Apreciando experiencias de países como Estados Unidos y Japón, Colombia podría apropiar espacios donde al emprendedor se le permita errar sin que eso signifique el fin de sus proyectos. Lo anterior, implicaría que se destinen fondos suficientes para apoyar y fortalecer las políticas públicas y el marco normativo actual, a fin de impulsar procesos innovadores concordantes con el contexto global facilitando, además, que puedan generarse fuentes de empleos sostenibles, con mediación de componentes como la investigación y las tecnologías, bajo un escenario socio-económico ajustado a las exigencias de la economía globalizada.

\section{Referencias}

Abdala, M. E. (2018). Análisis crítico del proyecto de ley de creación de las sociedades por acciones simplificadas. Revista Prolegómenos. Derechos y Valores de la Facultad de Derecho, 21(41), 163-177.

Aleksejeva, L. (2016). Country's competitiveness and sustainability: higher education impact. Journal of Security \& Sustainability Issues, 5(3).

Asamblea Nacional Constituyente (1991). Constitución Política de Colombia (1991), recuperada de: http:// www.secretariasenado.gov.co/senado/basedoc/constitucion_politica_1991.html

Baquero, Y. P. C., Hernández, J. S. R. y Saboga, J. F. G. (2019). La Formación de Emprendedores Sociales desde Algunos Programas de Contaduría Pública. In Vestigium Ire, 12(1), 165-185. 
Bohórquez, K. (2020). Tasa de desempleo en Colombia se ubicó en 9,7\% en 2018. La República. Recuperado de: https://www.larepublica.co/economia/desempleo-en-colombia-en-2018-2822565

Boutillier, S. \& Uzunidis, D. (2014). The theory of the entrepreneur: from heroic to socialised entrepreneurship. Journal of Innovation Economics Management, (2), 9-40.

Cáceres, N. D. (2017). La creación de valor compartido: estrategia de sostenibilidad y desarrollo empresarial. Cultura Latinoamericana, 22(2), 207-230.

Castro, M. A. B., García, M. L. S. y Adame, M. E. C. (2015). Hacia una comprensión de los conceptos de emprendedores y empresarios. Suma de negocios, 6(13), 98-107.

Chohan, U. W. (2019). Documentary Research: Positing Innovations in a National Budget Process. London: SAGE.

Código de Comercio de Colombia (1971). Código y disposiciones generales para el comercio en el país. Disponible en: http://www.secretariasenado.gov.co/senado/basedoc/codigo_comercio.html

Congreso de la República (2003). Decreto 934 de 2003 - por el cual se reglamenta el funcionamiento del Fondo Emprender - FE. Disponible en: https://diario-oficial.vlex.com.co/vid/decreto-354178578

Congreso de la República (2004). Ley 905 de 2004 - Promoción de la pequeña y mediana empresa. Disponible en: https://www.forpo.gov.co/es/normatividad/leyes/leyes-2004/179-ley-905-de-2004/file

Congreso de la República (2006). Ley 1014 de 2006, fomento a la cultura del emprendimiento. Disponible en: http://www.secretariasenado.gov.co/senado/basedoc/ley_1014_2006.html

Congreso de la República (2008). Ley 1258 de 2008, creación de sociedad por acciones simplificada. Disponible en: http://www.secretariasenado.gov.co/senado/basedoc/ley_1258_2008.html

Congreso de la República de Colombia (1996). Ley 344 de 1996, recuperada de: https://www.redjurista.com/ Documents/ley_344_de_1996_congreso_de_la_republica.aspx\#/

Congreso de la República de Colombia (1999). Ley 550 de 1999. Disponible en: https://colaboracion.dnp.gov. co/CDT/Desarrollo\%20Territorial/La_Ley_550_de_1999.pdf

Congreso de la República de Colombia (2002). Ley 789 de 2002, disponible en: https://sintesis.colombiacompra.gov.co/normatividad/documento/14144

Congreso de la República de Colombia. (2006). Ley 1014 de 2006. Artículo 1. Definiciones, Diario Oficial No. 46.164.

Correa Assmus, G. (2019). Innovación y emprendimiento. Ámbito Investigativo, 4(3), 62-65.

Corte Constitucional de Colombia (2005). Sentencia C - 448 de 2005, autonomía de entidades territoriales en materia tributaria-Competencia de asambleas departamentales y concejos distritales y municipales para determinar los elementos del tributo no fijados en la ley y las condiciones específicas en que operará. Disponible en: https://www.corteconstitucional.gov.co/relatoria/2005/C-448-05.htm

Corte Constitucional de Colombia (2007). Sentencia C - 392 de 2007, sociedades en ley de fomento a la cultura del emprendimiento. Disponible en: https://www.corteconstitucional.gov.co/relatoria/2007/C-392-07. htm

Creswell, J. W., \& Creswell, J. D. (2017). Research design: Qualitative, quantitative, and mixed methods approaches. London: SAGE.

Dälken, F. (2014). Are Porter's five competitive forces still applicable? A critical examination concerning the relevance for today's business (Bachelor's thesis, University of Twente).

DANE. (2020). Gran encuesta integrada de hogares, mercado laboral. Recuperado de: https://www.dane.gov. co/index.php/estadisticas-por-tema/mercado-laboral/empleo-y-desempleo 
DNP. (2019). Panorama laboral a agosto de 2019. Recuperado de: https://colaboracion.dnp.gov.co/CDT/Desarrollo\%20Social/Mercado\%20Laboral\%20agosto\%202019.pdf

Durango, I. C. M. y Rueda, C. A. J. (2020). Competitividad y productividad del administrador de empresas en las PYMES en Colombia y Latinoamérica. Tendencias, 21(1), 238-251.

Estrada, J. y Uribe, A. (2013). Análisis del emprendimiento en las principales ciudades de Colombia. (Trabajo de grado). CESA, Bogotá.

Gonzalez-Brambila, C., Jenkins, M., \& Lloret, A. (2016). Challenges for scholarly business research in Latin America. Journal of Business Research, 69(2), 383-387.

Guerrero, M., Urbano, D. y Fayolle, A. (2016). Entrepreneurial activity and regional competitiveness: evidence from European entrepreneurial universities. The Journal of Technology Transfer, 41(1), 105-131.

Guerrero, M., Urbano, D., \& Herrera, F. (2019). Innovation practices in emerging economies: Do university partnerships matter? The Journal of Technology Transfer, 44(2), 615-646.

Gutiérrez, J. G. y Baquero, J. E. G. (2017). New cross-proposal entrepreneurship and innovation in educational programs in third level (tertiary) education. Contaduría y Administración, 62(1), 239-261.

Kraus, S., Niemand, T., Halberstadt, J., Shaw, E., \& Syrjä, P. (2017). Social entrepreneurship orientation: development of a measurement scale. International Journal of Entrepreneurial Behavior \& Research.

Krauss Delorme, C., Bonomo Odizzio, A. y Volfovicz León, R. (2018). Modelo predictivo de la intención emprendedora universitaria en Latinoamérica. Journal of technology management \& innovation, 13(4), 84-93.

Marshall A. (1890). Principles of economics (9.a), MacMillan, London.

Melo, L. V. y Villa, L. (2015). Panorama actual de la innovación social en Colombia. Editado por el Banco Interamericano de Desarrollo (BID). Disponible en: https://www.iadb.org/en

Mintrabajo. (2019). "MiPymes representan más de $90 \%$ del sector productivo nacional y generan el $80 \%$ del empleo en Colombia". Recuperado de: Arangohttps://www.mintrabajo.gov.co/web/guest/prensa/ comunicados/2019/septiembre/mipymes-representan-mas-de-90-del-sector-productivo-nacional-y-generan-el-80-del-empleo-en-colombia-ministra-alicia-arango

Mok, K. H. (2015). Higher education transformations for global competitiveness: Policy responses, social consequences and impact on the academic profession in Asia. Higher Education Policy, 28(1), 1-15.

Molano, C. G. y Campo, J. L. M. (2014). Gerencia estratégica e innovación empresarial: referentes conceptuales. Dimensión empresarial, 12(2), 107-116.

Neto, RDCA, Rodrigues, VP y Panzer, S. (2017). Explorando la relación entre el comportamiento emprendedor y la satisfacción laboral de los docentes. Enseñanza y formación docente , 63 , 254-262.

Palma, H. H., Rojas, D. M. y Barrios I. (2017). Estilos gerenciales y su influencia en la generación de valor de las Instituciones Prestadoras de Salud de la Región Caribe. Económicas CUC, 38(1), 133-146.

Pazmiño, D. C. (2019). Comparando sistemas de innovación: una revisión de la literatura sobre Alemania y China. Revista Economía y Política, (29), 87-104.

Pinzón, L. D. B. (2019). Internacionalización y competitividad. Revista Ciencias Estratégicas, 22(32), 187-196.

Porter, M. E. (1979). How competitive forces shape strategy (pp. 21-38). Harvard Business Review.

Porter, M.E. (1990). The Competitive Advantage of Nations. New York: The Free Press

Rangel, P. E. S., Rubiano, M. E. M. y Riaga, C. O. (2015). Interacción universidad y entorno: marco para el emprendimiento. Educación y Educadores, 18(1), 2.

Reníz-García, J. R. y Rojas-Millán, L. K. (2018). Emprendimiento e innovación para impulsar la competitividad 
desde la universidad en la ciudad de Barranquilla (Colombia). Revista Espacios, vol. 39 (44).

Rivera, P. L. G. y Rodríguez, D. H. (2015). Educación para el emprendimiento. Mendive, 13(4), 418-423.

Rubach, M. J., Bradley III, D., \& Kluck, N. (2015). Necessity entrepreneurship: a Latin American study. International Journal of Entrepreneurship, 19, 126.

Rubiano Riaño, C. A. (2020). Revisión de los factores que limitan la innovación en las empresas colombianas. Proyecto de Investigación UNAD, recuperado de: https://repository.unad.edu.co/handle/10596/31798

Salcedo-Pérez, C., Moscoso-Duran, F. F. y Ramírez-Salazar, M. P. (2020). Economía informal en Colombia: iniciativas y propuestas para reducir su tamaño. Economía, 41(03).

Sánchez García, J. C., Ward, A., Hernández, B. y Florez, J. L. (2017). Educación emprendedora: Estado del arte. Propósitos y Representaciones, 5(2), 401-473.

Schumpeter, J. A. (1934). Change and the Entrepreneur. Essays of JA Schumpeter.

Silva, J. M. Á. y Bermúdez, G. M. (2020). Obligaciones de los Estados respecto de la protección de los derechos humanos: en el trabajo y la seguridad social. Justicia, 25(37), 1-26.

Sölvell, Ö. (2015). The Competitive Advantage of Nations 25 years-opening up new perspectives on competitiveness. Competitiveness Review, 25(5), 471-481.

Tamayo, E. F. S. y Alvear, R. F. H. (2018, April). El emprendimiento y sus factores de éxito ligados a la formación universitaria: un análisis de los casos generados por profesionales egresados de las facultades de administración de Guayaquil-Ecuador. In [2018] Congreso Internacional de Ciencias Sociales.

Taylor, S. J., Bogdan, R., \& DeVault, M. (2015). Introduction to qualitative research methods: A guidebook.

Torres-Valdés, R. M. (2015). Estímulo a la creatividad y emprendimiento joven para la innovación social en un contexto global. Universidad de Alicante. Departamento de Comunicación y Psicología Social. Disponible en: http://hdl.handle.net/10045/53448

Trujillo Murcia, I. G. y Estrada Orozco, Y. L. (2017). Las asociaciones por acciones simplificadas SAS (Bachelor's thesis, Universidad de Bogotá Jorge Tadeo Lozano).

Vega Guerrero, J. C. y Mera Rodríguez, C. W. (2016). Modelo de formación en emprendimiento social para Instituciones de Educación Superior en Colombia. Revista EAN, (81), 29-44.

Villarreal, A. F. S. (2017). ¿La SAS el vehículo comercial por preferencia? Revista Cultural Unilibre, (2), 14-29.

Wang, H. (2014). Teorías para la ventaja competitiva. En H. Hasan (Eds.), Being Practical with Theory: A Window into Business Research (pp. 33-43). Wollongong, Australia: THEORI. http://eurekaconnection.files. wordpress.com/2014/02/p-33-43-theories-of-competitive-advantage-theori-ebook_finaljan2014-v3. pdf 
